different types of evidence - and the appropriate dose-response models which could threfore be justified.

Five members of the committee issued a dissenting statement, claiming that insufficient weight had been given to radiobiological data and that accepting the linear hypothesis as a basis for regulation, would result in regulations which would hinder the development of nuclear energy and the use of radiation in medicine.

When it became clear that additional members of the committee also had reservations, academy president Dr Philip Handler decided that the controversial section should be rewritten. The revised report was published in Washington last week, with dissenting statements from Drs Radford and Rossi.

The new report goes some way to establishing a compromise on what the academy calls the "ideological differences" between committee members over methods for estimating numerical risk values for cancer induction.

Whereas previously, for example, the committee had used data on general cancer incidence taken from survivors of the Nagasaki atomic bomb explosion, the new report gives this data less prominence than the cancer mortality data on survivors in both Hiroshima and Nagasaki, which suggests a more quadratic dose-response relationship at low radiation levels.

Similary, rather than giving estimates of predicted increased cancer mortality and morbidity based directly on the linear hypothesis for the effects of low LET (linear energy transfer) radiation primarily $\mathrm{X}$-rays and gamma rays - the new report presents a range of risk estimates based on linear, linear-quadratic and quadratic models. And it adds that the linear-quadratic is "most consistent with existing human epidemiological studies and radio-biological data and knowledge",.

Dr Radford took no part in the rewriting process. He remains critical of the final results, claiming that the report ignores much new data in support of the linear hypothesis, with the consequent risk of understating the dangers of radiation.

Dr Rossi, too, remains dissatisfied. $\mathrm{He}$ claims that his own work and that of others on the effects of radiation on plant cells suggests that the linar hypothesis overstates the dangers. And that even though its prominence has been downplayed, it is still likely to be used in standard setting, to the disadvantage of the nuclear industry.

David Dickson

\title{
Calculations and measurements
}

What follows is a summary of the definitions in BEIR III relating to the somatic effects of ionizing radiation. The terms of reference of the study, set by the US Environmental Protection Agency, include an explicit reference to nonlinear effects. The report therefore includes a discussion of the small-scale effects of the interaction of radiation with matter along the following lines.

Radiation dose $(D)$ is a quantity of energy deposited in matter; the unit is the rad (equivalent to 100 ergs of absorbed energy per gramme), soon to be replaced by the SI equivalent of the gray (or Gy), with 1 $\mathrm{Gy}=100 \mathrm{rad}$. Charged particles (e.g. $\alpha$ particles) or uncharged and indirectly ionizing particles (e.g. X rays) differ from each other in the rate of "linear energy transfer" (LET) along their tracks; for a given particle, the LET is also a function of energy. The LET is low (a few keV per $\mu \mathrm{m}$ ) for cosmic-ray $\mu$-musons, high for $\alpha$-particles.

Concerned as it is with nonlinear effects, BEIR III needs to introduce a microscopic analogue of the macroscopic dose $D$ called "specific energy" $z$ and intended as a measure of energy deposition by radiation in sub-cellular structures such as cell nuclei.

The deposition of energy along the track of a particle is not uniform on such a scale, because the interactions of radiation with matter entail interactions with discrete entities in the irradiated matter. Thus although (with appropriate units) the average value of $z$ (written $\bar{z}$ ) must equal $D$, the microscopic intensity of energy deposition can vary dramatically with different kinds of radiation.

The report goes on to consider (but does not eventually adopt as an assumption) the proposition that for radiation to cause somatic damage, two "sublesions" must occur within some small range (say $1 \mu \mathrm{m}$ ) in a cell and within some specified interval of time. Then the frequency of "lesions" $(E)$ will be $K\left(\overline{z^{2}}\right)$ where $K$ is a constant. Moreover, the mean square microscopic dose will be related to the macroscopic dose $(D)$ by $\overline{z^{2}}=w D+D^{2}$ where $w$ is a constant determined unambiguously by the statistical properties of $z$ for the type of radiation concerned.

One consequence of this argument is that even the assumption that it takes two sublesions ("hits") within some microscopic region leads to a dose-effect relationship in which the risk of damage is of the form $\beta D+\gamma D^{2}$, but in that case $\beta$ and $\gamma$ should be related to each other by the statistical properties of the radiation concerned, whence the relative biological effectiveness of high-LET and low-LET radiation should be calculable.

With this said, BEIR III says that wherever possible empirical data have been fitted to curves with the form $\left(a_{0}+\alpha_{1} D+\alpha_{2} D^{2}\right) \exp \left(-\beta D-\beta_{2} D^{2}\right)$ where all the constants are positive, $a_{0}$ represents the spontaneous rate of some effect and the exponential term (significant only at high doses) takes account of competition from cell death.

BEIR III includes a discussion of using human epidemiological data as a means of determining dose-response relationships empirically. Apart from familiar problems of bias (people exposed to diagnostic $\mathrm{X}$-rays may be especially prone to cancer, for example), the report draws attention to the retrospective character of most epidemiological studies, the problems occasioned by the long latency period for cancer induction, the distinction between mortality and morbidity (and the possibility that the latter may be underreported), differences of susceptibility between different genetic groups.

Numerical calculations of cancer caused by radiation yields rates per million people assumed to be taken from a population represented by life-tables for the United States population in the period $1969-71$.
The BEIR report uses two alternative models for calculating the "excess" effects of radiation. Absolute risks from radiation exposure are represented by constant probabilities of cancer formation, or of death therefrom, for each year of life after the lapse of the latency period. Relative risks are calculated on the assumption that the effect of radiation exposure is to increase the formation of cancer, or of cancer mortality, in proportion to the spontaneous rate at the age at which the damage becomes apparent.

Like previous analyses of the consequences of radiation exposure, BEIR III leans heavily on data gathered over the years from the populations of Hiroshima and Nagasaki, but again it breaks new ground by seeking to separate the effects of gamma and neutron doses at the two explosions. The two explosions differed in that at Nagasaki the explosion exposed the population to much smaller neutron doses than that at Hiroshima.

To make the calculations of risk explicit, BEIR III considers three separate cases of radiation exposure: (a) a single exposure of a population represented by the US lifetables to 10 rad of low-LET radiation; (b) a continuous lifetime exposure at a rate of 1 rad per year; and (c) a pattern of exposure to $1 \mathrm{rad}$ a year at ages chosen so as to simulate occupational exposure to radiation.

Different assumptions give very different results. Spontaneous cancer mortality for the standard US population is calculated at 16.38 per cent, and the consequences of a single exposure of the whole population to a dose of $10 \mathrm{rad}$ turns out to be an extra 766 deaths $(0.47$ per cent $)$ on the absolute risk calculation and an extra 2,255 deaths ( 1.4 per cent) on the relative risk basis, on the assumption that the doseeffect relationship is represented by a quadratic function including a linear term

BEIR III is so far distributed in typescript form, but will be available in book form from the National Academy of Sciences later in 1980. 